formed regarding it), the nervous impulses (in most normal individuals and in ordinary circumstances, at all events) pass to and through the auditory speech centre. Further, it seems probable, so far as our present knowledge enables us to judge, that (in most normal individuals and in ordinary circumstances at least) the emissive impulses (after the answer has been decided upon) first pass to the auditory speech centre (where they are, as it were, first put into concrete speech in the form of auditory speech symbols), and then flow to the visual speech centre, where they are translated into visual speech symbols, from which they pass to the graphic (? speech) centre, from which they are emitted (see Figs. 4 and 5). But whether the passage from the visual speech centre to the emissive writing or graphic (? speech) centre is a direct passage, as I believe and as Dr. Bastian has argued (and as is represented in Fig. 5), or whether these emissive impulses must first pass to and through the rocal speech centre (Broca's centre), as many authorities suppose (as is represented by the dotted lines (Vi.S.C. to Vo.S.C. and Vo.S.C. to G.S.O.) in Fig 6), is still a matter of uncertainty.

\section{FIG. 6.}

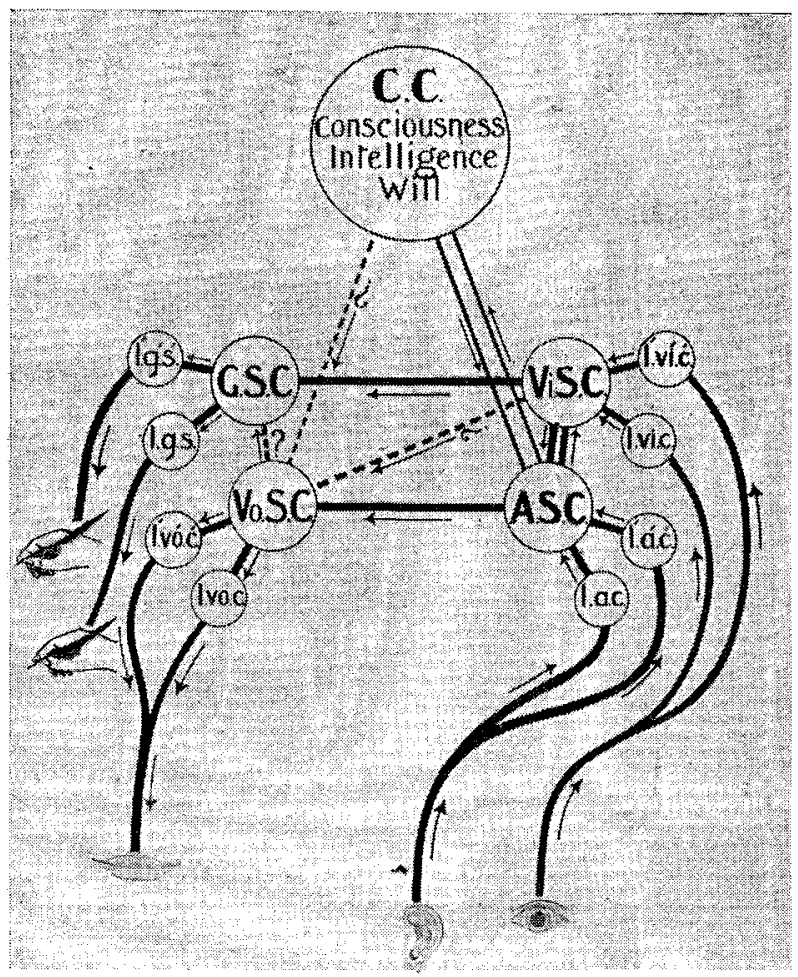

Diagrammatic representation of the course taken by the nervous impulses concerned in the production of (1) a spoken answer to a spoken question; (2) a written answer to a spoken question; (3) a spoken answer to a written question; and (4) a written answer to a written question. The meaning;of the letters and centres has been explained under Figs. 2, 3, 4, and 5 .

That the course of the nervous impulses concerned in the complicated cerebral processes to which I have just referred is such as I have described is chiefly based upon evidence derived from two sources-viz.: (1) the clinical and pathological observation of cases of aphasia; and (2) the study of the order and manner in which the speech faculties are trained and developed in the normal child.

It is important to remember that the derangements of speech and intellectual language included under the term aphasia are degradations of the speech functions produced by disease. In other words, they are the result of a process of dissolution, whereas the development and training of the speech mechanisms and functions are the result of a process of evolution, and that by contrasting the different forms of speech derangements which result from disease with the different stages of speech development in the normal child our knowledge of the pathological physiology, as I term it, of aphasia is greatly assisted. Further, from a study of the development of speech in the young child we see the manner in which aphasic cases should be treated, the manner in which the speech mechanism and speech faculties after they have been destroyed or deranged by the process of dissolution produced by disease should be re-educated and re-acquired.

\section{PROFESSOR A. E. WRIGHT'S METHOD OF TREATING TUBERCULOSIS.}

BY W. WA'TSON CHEYNE, C.B., LL.D., F.R.S., F.R.C.S. ENG.,

SENIOR SURGEON TO KING'S COLLEGE HOSPITAL, ETC.

I REGRET that I was not able to be present at the meeting of the Royal Medical and Chirargical Society at which Professor A. E. Wright's paper on the treatment of tuberculosis was discussed, but as he and his colleagues have drawn some far-reaching clinical deductions from their work I should like to indicate how the matter strikes me from the standpoint of the practising surgeon. Whatever view we may take of the position which tuberculin should occupy in the treatment of local tuberculoses I think we must all agree that Professor Wright has done a piece of fine, original, scientific work and that he has apparently indicated an important guide in our combat with infective diseases in the shape of what he terms the "opsonic index." But we must remember that, though from time to time Professor Wright speaks of his conclusions as new facts, we are really not dealing with facts but with theories-with excellent working hypotheses, no doubt, but still with theories-which are certain to undergo considerable modification as the investigation goes on and experience accumulates, Hence we must proceed cautiously when we come to translate these theories into practice and we must not violently throw over all our past clinical experience in favour of views which will almost certainly require modification and expansion before long.

The root of the matter is the question of opsonins and the opsonic index, and as regards the latter the points are it accuracy, its significance, and its value in determining the effect of treatment. I shall merely touch on these points. If I understand Professor Wright aright his view is that the mode in which the body defends itself against bacteric invasion is by the development in the blood serum of substances termed opsonins, each bacterium leading to the development of its special opsonin, which coming in contact with the living bacteria weaken them and enable the phago cytes to complete their destruction. Apparently the body has no other means of defending itself and the only way of aiding recovery in cases of tuberculosis, for example, is to try to increase the opsonic power in the blood. This is done by introducing minute quantities of dead bacteria of the same kind as those invading the body and securing that the blood serum comes in contact with the invading organisms. The theory is deliciously simple, but I doubt if any of the minute processes of the body can be explained in such a simple way. I think I may say that in all cases where a simple theory like the above has been enunciated it has been found necessary to modify and enlarge it till the matter has been found to be of the greatest complexity. Many reasons might be adduced against the simplicity of this view but I do not propose to discuss this matter fully and shal merely indicate some points which make me doubt its completeness.

As to the accuracy of the opsonic index I do not presume to judge, but it has struck me from what $I$ have seen that the result of the examination of the blood depends a good deal on the personal equation and has not the strict mathematical accuracy that one could desire. Individuals will differ in the way in which they will prepare the dilutions, more especially of the bacilli, and so on. Nevertheless, judging from the reports there is a remarkable correspondence in the results obtained by different observers. The chief difficulty seems to be in the cumbersomeness of the method which pats it outside the power of the busy practitioner and if this method is to hold its own-which may or may not be the case-the various clinical laboratories would need to make arrangements for examining and reporting on specimens of blood sent to them, and at moderate rates.

When we come to the significance of the opsonic index I confess to being somewhat puzzled by the statements of different observers and I begin to donbt whether Professor Wright's views really express the whole truth in the matter Apparently a distinction arises between the results which are obtained in cases of strictly localised tubercle, such as lupus, and in the more generalised and acute forms and it has evidently required considerable ingenuity to devise theories 
which will reconcile these results. So far as I gather, Professor Wright and his colleagues apparently look on a tuberculo-opsonic index which is distinctly below the normal standard (spoken of as 1) as indicating tuberculosis, or at any rate a condition in which the patient is very liable to become tuberculous; and if this were always the case one would have little hesitation in accepting this view. Apparently, however, there are many exceptions. For example, Dr. W. Bulloch found in the case of lupus that no less than 25 per cent. were above the lower normal limit of 0.8 , and something like 10 per cent, were at or above the normal standard of healthy people--namely, 1 . No doubt a number of these cases had been under treatment in various ways and the treatment may have modified the index, but 25 per cent. is a very large proportion of exceptions to a law and raises my suspicion that this law does not express the whole truth. Again, in chronic phthisis 10 out of 25 showed an index at or above 0.8 , as stated by Dr. D. Lawson and Dr. I. S. Stewart, while Dr. G. W. Ross states that in 100 cases in the wards of Victoria Park Hospital-presumably many of them tuberculous cases-51 had an index above $1 \cdot 2,38$ between 0.8 and 1.2 , and only 11 below. Apparently in chronic cases of tuberculosis the index is as a rule below normal, while in the more acute cases the index, though fluctuating, is often above the normal. Hence the conclusion is drawn that a tuberculo opsonic index either below or above the normal-that is, below 0.8 or above 1.2 -indicates tuberculosis. Surely here we have a straining of theory to meet the facts. Professor Wright apparently explains the high index in the more acute cases by supposing that it is due to auto-intoxication and as these patients do not do well he looks upon these inoculations as being badly adjusted and interspaced. But what puzzles me is that Professor Wright at the commencement of his paper showed that too large or too frequently repeated doses of tuberculin lead to a cumulative effect in the direction of the negative phase, and I should have expected that in these acute cases, where presumably on the view of auto-inoculation the doses were excessive, or at any rate too frequently repeated, an extreme negative phase would have been induced and the opsonic index would have stood far below the normal. And where in these cases the contrary was the case one would have expected them to show marked improvement in correspondence with the high index. It seems to me that something is wanting in the theory to account for these discrepancies.

Professor Wright also states that "We are, I think, warranted in conceiving of the low opsonic power which is found in association with strictly localised infections as a condition which dates back to a period anterior to infection." of this statement I cannot find any proof and I note that one at least of his colleagues disagrees with him. It may be correct, but it is also possible that this low opsonic index is only found when infection is present and that it is the existence of the local lesion which keeps down the index. We must remember that bacteria produce substances which are deleterious to the body and which presumably in that way aid their own spread, and it may be the absorption of these products which affects the opsonic index. Professor Wright points out that even after imperfect surgical operations the index alters and he apparently thinks that this means that during the operation bacilli have been forced into the blood stream ; in other words, the patient has had a dose of tuberculin and that this has affected the index. May the explanation not, however, be that the rise of the opsonic index after these surgical operations was due to the removal of the greater part, at any rate, of the substances which were entering the blood and depressing the index? And may it not be that the rise of the index during the course of the disease does not imply an auto-inoculation, as Professor Wright assumes, but a cessation of the production or absorption of the products of the bacilli which have been producing the low index? This matter does not appear to me one of any special importance apart from the statement with regard to the effect of surgical operations. But it further illustrates the difficulties in connexion with the opsonic index theory in its present condition.

The next point to which I would refer is the value of trberculin in tuberculous disease. I have from the very first looked on tuberculin, even the old tuberculin, as a valuable adjunct in the treatment of tuberculosis, and in former years I had cases-few, it is true-where patients have remained well after a prolonged course of treatment. And the conclusion which $I$ came to at that time was that while in surgical cases where other and safer means were at hand for dealing with the disease the sphere of tuberculin was somewhat restricted, yet in cases which were not amenable to surgical treatment it still remained a valuable adjunct. It has always been a matter of great surprise to me to notice the way in which the treatment was suddenly thrown on one side simply because it did not come up to the expectations which had been formed of it and because in some cases the results were disastrous. I have always felt. that if I had been a physician I should have continued to work with tuberculin, feeling that thorough investigation as to the best method of using it would have overcome many of the objections. Hence I am delighted to find that the result of Professor Wright's work is to testify to the excellence of Koch's discovery, and that as the result of the former's method of administering tuberculin Koch's discovery may be replaced on its proper footing. At the same time, assuming that Professor Wright's theory is complete and that the effect is due to raising the opsonic index, I do not know that I feel altogether satisfied that in tuberculin we have an absolutely perfect means of doing so. Nor do I see sufficient proof to enable us to say that if only the opsonic index can be kept up the bacilli will ultimately disappear and the patient be cured. Indeed, the presence of a normal index does not seem at all inconsistent with the growth of the bacilli. That tuberculin is the best means at our hand at present I am quite willing to admit but that other means may before long be discovered is, I think, highly probable. In any case my feeling is that it is not sufficiently certain to produce a cure to warrant us in disregarding and throwing aside other methods of treatment. which have up to now given fairly satisfactory results.

In former papers Professor Wright has pointed out the importance of a flow of lymph through the focus of an infective inflammation, especially in connexion with abscess and tuberculous peritonitis, and long ago I suggested exactly the same explanation of the improvement which followed surgical treatment in those cases. It was one of the early clinical facts pointed out by Lord Lister when he began to work at antiseptic surgery that if a well-formed acute abscess be opened and drained by means of a drainage-tube, and if every care be taken to prevent tresh infection from without, suppuration at once ceases. On the following day the discharge will be found to have been copious, but to be essentially serous, and the cavity rapidly closes without further suppuration. The explanation which I gave was that after the opening of the abscess large quantities of serum oozed through the granulation tissue in which the bacteria were situated and that this serum contained antibacterial substances which killed, or at any rate weakened, the cocci to such an extent that they could not resist the destructive action of the tissues or phagocytes. Professor Wright has given the same explanation practically in the same words. But I have always doubted whether this was really the whole explanation. For example, if the incision be made at an early period when suppuration is only beginning pus formation does not cease as it does when the abscess has been, so to speak, well formed. This may be because at the early period sufficient antibacterial substances have not yet accumulated in the serum of the blood. But, on the other hand, that may not be the whole explanation and it raises the question in my mind whether besides phagocytosis there are not other local factors which take an important part in the destruction of bacteria and which are not ready to take up this function if incisions are made at too early a period of the inflammation.

Similarly the effect of merely opening the abdomen in cases of tuberculous peritonitis is in some cases very remarkable, and although physicians have of late thrown doubts on the value of surgical interference in these cases I think that most surgeons who have had experience of them will not share that view. The only explanation which I could think of for the improvement after simple opening and closure of the abdomen was that serum containing antibacterial substances was poured out after the operation and interfered with the vitality of the tubercle bacilli. But here again I can hardly believe in my own explanation. Improvement is seen in those cases where the abdomen is full of nodules and where the amount of serum which would be presumably poured out as the result of the comparatively slight surgical operation must be so small that one can hardly believe that it has soaked into the interior of these nodules and injured the bacilli which are contained in them. And again we have the clinical fact that aspiration in cases 
of tuberculous peritonitis, where a large quantity of ascitic fluid is present, does not have the same beneficial effect as a simple incision, even although in the latter case no subsequent drainage is employed. And here again, as in acute abscess, we find that if the operation is performed early in the case we do not have the same good results as if it is done at a later period. And, just as I doubt my own explanation, I confess again that I do not feel that Professor Wright's views represent the final and complete solution of the mode in which the invasion of bacteria is opposed by the body. My own feeling is that leucocytes and opsonins are not the only defensive agents of the body and that other factors, as yet unknown, are also of great importance. As regards leucocytes we know that in tubercles the bacilli are present in the giant and epithelioid cells and it is quite useless to look for them among the leucocytes.

A third statement made by Professor Wright must also be touched on as he draws extremely important and far-reaching conclusions from it as regards treatment on the assumption that his theory is a fact. $\mathrm{He}$ explains the persistence of local infections by supposing that the lymph finds difficulty in gaining access to the whole of the tuberculous deposit and that the antibacterial substances in it may be used up at the outer part of the focus, while the interior is not subjected to their influence. $\mathrm{He}$ further assumes that in localised infections there is deficient circulation, especially of lymph, and then he draws the deduction that, as part of the treatment, means must be taken to increase the flow of blood and lymph through the part. Now this view that there is deficient circulation of blood and lymph in localised infections is more or less an assumption and $\mathrm{I}$ should doubt if it were quite accurate in many instances. And especially in the case of tuberculosis, so long as we have to deal with isolated tubercles, and so long as a mass has not formed which is undergoing caseation in the centre, I should think there would be no difficulty in serum. soaking through the tubercle without any artificial aid. Here $I$ believe that the persistence of the local deposits depends as much on the weapons wielded by the bacteria as on the absence of antibacterial substances on the part of the body. Let us consider for a moment the question of the dosage of bacteria, which I demonstrated as an important scientific fact a good many years ago. In some instances I found that one or a few bacteria would suffice to infect the animal and as a rule in these cases the bacteria flourish in the blood stream rather than in the tissues. In others, again, I found that bacteria could not establish themselves in the body unless a large number were introduced at one part. The explanation which I gave of this was that the bacteria have weapons as well as the boay and that these weapons are not confined to the protoplasm of the bacteria but are poured into the surrounding tissues, lower their vitality, and enable the organisms to establish themselves, so that, where the body is not particularly susceptible, a large number of bacteria must act together in order to obtain a footing. And I believe that the persistence of a local deposit is not due in all, or indeed in many, cases to difficulty of entrance of the body fluids but to other causes among which may be mentioned the number of bacteria and their products in the deposit, and I very much doubt if attempts to increase the activity of the circulation, even if loaded with opsonins, in these parts are at all wise from a clinical point of view.

In connexion with this matter Professor Wright states that " the infecting organisms cultivate themselves in the organism in every case in a region of lower bacterio-tropic pressure-that is, in a region where the anti-bacterial substances are either absent or, at any rate, greatly reduced in amount as compared with what is found in the circulating blood." This view is very difficult to reconcile with what we know as to the seat of tuberculous lesions. We can hardly imagine that before a tuberculous lesion is established there are parts in the body in which the serum and the circulating blood lose their opsonins, and therefore it is difficult to assume that it is the absence of opsonins in a particular part that enables the bacteria to establish themselves there. As a matter of fact, as regards tubercle, the disease very often establishes itself in parts which are particularly highly vascularised. For example, in bone tubercle the favourite seat is the neighbourhood of the epiphyseal line where, as a matter of fact, the vascularity is greatest; and in lupus the favourite seat is the nose and face where also there is very marked vascularity. Then, ag tin, apart from vascularity of the part, other local reasons must exist to explain why tubercle chooses one part rathe than another-e.g., Why is hip-joint disease so very much more common than disease of the shoulder-joint, and so on? Hence I should say that there are clearly local factors quit apart from the absence of opsonin at work in the establish. ment of the tuberculous focus and I see no reason why these local factors may not continue at work in maintaining the existence of that focus.

To sum up, the whole of these considerations incline me to doubt whether in opsonins and phagocytes we have the whole defensive armamentarium of the body against tubercle bacilli and whether there are not many other factors, especially local factors, which are of im. portance, and perhaps of equal value, in connexion with the maintenance and spread of the disease. And hence my doubt whether it is wise in connexion with the treat. ment to devote our whole attention to one point-namely, the raising of the opsonic index, and to neglect other points which may be also of essential importance. I shall not, however, discuss the scientific aspects of Professor Wright's paper any further but will now come to the clinical applica. tion of his theories. It seems to me that Professor Wright has not been fortunate in his clinical experience, especially as regards the value of surgery in the treatment of tuberculosis. And more especially when he speaks of the results of radical operations I doubt if the cases he quotes could be classed as really radical.

In the first place, he makes the statement that strictly "localised infections do not tend to get well. They are characterised by an altogether indefinite duration." Now this statement is quite inaccurate. It is true with regard to lupus that even under the very best hygienic treatment without some surgical or medical interference there is very little tendency for the disease to cease; but even in lupus patches disappear without treatment, although no doubt extremely rarely, and one not uncommonly sees that the centre of a patch of lupus has healed while the disease is still spreading at the periphery. But lupus stands practically by itself among the localised tuberculous infections in its tendency to persist. I think I may say that in the case of every other localised tuberculous infection the disease has a strong tendency to get well even without operative treatment, if the patient is placed under suitable conditions. We know perfectly well that glands in the neck, diseases of joints, diseases of bones, diseases of the spine tuberculous peritonitis, \&c., will often recover apparently completely under suitable treatment; at any rate, as completely as the cases described by Professor Wright and his colleagues as the result of tuberculin treatment. And therefore I can only look on the use of tuberculin as an additional weapon in our hands; one, I believe, of the very greatest value when properly administered, but not as a substitute for well-established lines of treatment.

Another point to which I may refer is Professor Wright's view as to the surgeon's attitude to local tuberculosis. Apparently, in his opinion, the surgeon has no other thought in the treatment of these cases than extirpation and the application of antiseptics. Professor Wright says: "I would, in view of this new asset in medicine, fain induce the surgeon to abate something from his conviction that extirpation and the application of antiseptics offer, in connexion with bacterial infections, the only possible means of cure." This is quite a grotesque exaggeration. For, as a matter of fact, it is only in a comparatively small proportion of cases of localised tuberculosis that the surgeon resorts to operative means. It is not every case of glands in the neck, for example, which comes to a surgeon which is treated by extirpation. Certain cases evidently require operation; for example, those where the glands are breaking down and where pus is forming. But a very considerable number of cases of enlarged glands in the neck, which are in all probability tuberculous, are treated by the surgeon by other methods, especially by bygienic measures, before any operative interference is advised. This is $\mathbf{s}^{+}$ill more the case in connexion with diseases of bones and joints. The surgeon does not forthwith proceed to operate on a tuberculous joint nor does he consider that operation offers the only means of cure. It is only when he finds that the tuberculous disease resists other methods of treatment and where the case is going down-hill that he feels it necessary to interfere. And just as the surgeon knows that there are other methods of treatment besides operation, so $I$ think it is well for the pathologists to bear in mind that there are other methods of 
dealing with tuberculosis besides treatment by tuberculin, and if they blindly pursue the course of treating patients by tuberculin as the only method of treatment they will throw away many weapons which otherwise would be of advantage in dealing with the disease. Besides, if I may mention another clinical point, the object of surgical operations in tuberculosis is not always to remove the infected area and to extirpate the infection. There are various other considerations which have to be borne in mind in treating a case besides the removal of the disease. The situation of the disease may lead to consequences which may require treatment apart from its mere cure; and I hope that Professor Wright's view that the surgeon is not to be called in till tuberculin has failed will not become a generally accepted one, otherwise we shall have very curious results as regards deformities and other troubles which ought not to occur if one bears in mind the fact that the treatment of the disease by raising the opsonic index is not the only point to be considered.

With the view of assisting the action of tuberculin Professor Wright lays the greatest stress on the importance of increasing the flow of lymph through the affected part. I think that treatment directed in this way must be done with the greatest judgment. Professor Wright assumes quite wrongly that when a tuberculous sinus shows signs of drying up it means that it is not going to heal. Exactly the opposite is the case. As long as a tuberculous sinus is actively discharging, so long we know that for some reason or other the tuberculous process is active; but as the tuberculous disease becomes less active the sinus tends to dry up and in the course of time-very possibly it may be some months-provided no mixed infection occurs in the interim, the sinus will usually close. When Professor Wright leaves the question of tuberculin treatment and advises the employment of methods which increase the flow of lymph in various tuberculous lesions he is courting disaster. In such cases as psoas abscess, for example, the risk of sepsis will be increased and we know that the probability of recovery in cases of tuberculosis is very much greater where we have to do with a simple than with a mixed infection. I fear that if the surgeon employs substances which will produce a flow of lymph through the sinus he will very likely end by having a mixed instead of a single infection and then he will find that his tuberculin has much less curative power than it otherwise would have had. Similarly with the question of massage, movements, \&c., in promoting the flow of lymph through a tuberculous focus. We already know by ample experience that movement and massage are methods of treatment which are very likely to lead to serious exacerbation of the symptoms and increased activity of the disease. Even after the opsonic index has been reinforced by tuberculin injections the greatest care should, I think, be exercised in the employment of these measures.

Then, again, Professor Wright states that "it is one of the cherished beliefs of the surgeon that it is possible to extirpate completely and effectively by the knife in the large proportion of the cases in which he has to deal, the invading micro-organisms. I for my part find it very difficult indeed to believe that this result can often be achieved even by the most radical operations." This clinical observation is apparently based on operations in which the chief method of extirpation employed was scraping. I have for years inveighed against classing any operation in which scraping of a tuberculous lesion was employed as a radical operation. I quite admit that one cannot cure tuberculosis by radical operation, but clinical experience shows that in a large majority of suitable cases one can get rid of the particular local deposit by operation. I believe that a patient who has tuberculosis, say in a joint, has in all probability a deposit elsewhere in the body-in the bronchial glands it may be-which is unaffected by the operation on the local affection. But undoubtedly the operation on the local infection will in many cases, if really radically performed, cure that lesion. To amputate a limb well above the seat of a tuberculous lesion will surely be admitted to be an effective, though no doubt a very severe, method of getting rid of the disease. But many less drastic operations for the removal of the disease, if only one cuts sufficiently widely, are equally effective.

Take, for example, the case of glands in the neck to which Professor Wright especially refers. To scrape out glands in the neck may occasionally succeed, but in the great majority of cases it is an absolutely futile method of procedure. And even when it comes to excision I am afraid that many of the so-called radical operations on tuberculous glands simply consist in removing the glands which are visibly infected. Now that is not my idea of a radical operation for glands in the neck. It is quite certain that the infection extends beyond glands which are visibly enlarged and if only the latter are removed the condition is very prone to reappear. And therefore $I$ have always advocated an elaborate operation in cases of tuberculous glands in the neck, consisting not only in the removal of all infected glands but of all the fat and other glands in the neighbourhood over an extensive area. I believe it is advisable to remove the fat because we know that after the removal of glands the fat lobules very often develop lymphatic tissue and fresh glands are produced, and the very fact that in Professor Wright's experience recurrence has taken place at the seat of operation at once shows that the operation was not radical. I have had many cases of glands in the neck where a single operation has put a stop to the condition and where no recurrence whatever has taken place. But I frankly admit that in a good many cases recurrence has taken place but very seldom in the area of the operation. If the operation is performed as I pointed out above there is nothing for recurrence to take place in. Not only are all the glands, normal and infected, removed but the fat lobules, which might subsequently develop glandular tissue, are also taken away, and where recurrence has taken place it has been in other parts of the neck and simply implies that the operation has not been sufficiently extensive. For example, after operation on the anterior triangle the patient may come again with glands in the submaxillary triangle or beneath the chin or towards the lower part of the posterior triangle, but it is not my experience that the patient comes back with glands in the anterior triangle on which I have already operated and, as I say, in many cases where the operation has been thoroughly performed the patient has no further glandular trouble.

The same holds good with regard to other conditions. I do not know that there is anything more satisfactory than the excision of a small patch of lupus. If only the patch is quite small and the excision is carried out comparatively freely there may be no iurther appearance of the disease. I maintain that in certain bacterial diseases, more especially tuberculosis, the disease can be got rid of, as regards its local manifestations at any rate, by suitable operation, and even much more acute affections, such as antbrax pustules, cancrum oris, \&c., can often be effectually treated by surgical means and in them I would not advise trusting solely to attempts to raise the opsonic index. Even in bad pyogenic affections, such as carbuncle, I think that surgical treatment should be combined with Professor Wright's inoculations.

One other point which I note and which has made me doubt whether tuberculin, although an extremely valuable remedy, is the final and best result which will ever be obtained in the treatment of tuberculosis, is the fact that in spite of long-continued use of tuberculin and marked improvement in the patients, Professor Wright and his colleagues admit that tubercle bacilli may still be present in the urine on one hand in cases of urinary tuberculosis, in the sputum in cases of pulmonary tuberculosis, \&c., after a long period of treatment; and they also refer to recurrences taking place after the sores have healed in cases of tuberculosis on the surface of the body. Hence I do not feel that, as regards this question of recurrence and the eradication of the disease, we can absolutely depend on tuberculin.

I have, of course, nothing like Professor Wright's experience of tuberculin as employed by his method of dosage, but I have had several cases treated in this way, cases chiefly of bone and joint tuberculosis and of genito-urinary tuberculosis, and I must confess that I have not seen such remarkable improvement as Professor Wright's statements would lead one to expect. My view as regards the position of tuberculin in the treatment of tuberculosis is that it is a most valuable adjunct to our treatment. But I am not prepared to abandon surgical measures when they are necessary. Even in the case of glands in the neck I believe that cases which, apart from tuberculin, I wouid have operated on up to now still ought to be operated on. Glands which are breaking down, where pus is present, where ulceration has taken place, and so forth, are much better removed by a radical operation, and then by all means let the treatment be followed up by the use of tuberculin on Professor Wright's principle of dosage. Similarly in joint and bone diseases, where no operation would formerly or now have been considered necessary, by all means add the use of tuberculin to the other methods employed. But blindly to convert the 
practitioner into "an immunisator," as Professor Wright puts it, is, I believe, a totally retrograde step. I feel sure that unless care is taken, and if the same enthusiasm which animates Professor Wright, and which animated many of us in the days of the old tuberculin, is again excited, the result will be disappointment and very possibly again the throwing over of a most useful remedy in the treatment of tuberculosis. It is for this reason that I have thought it well to enter a protest against extravagance in views and the rash throwing away of many years of careful clinical observation and experience.

Harley-street, W.

\section{A MEDLEY OF SURGERY.}

\section{By Professor E. E. GOLDMANN.}

(Concluded from p. 2.4.)

\section{Conservative Operations for Malignant Growths} OF LONG BONES.

JUDGING from various publications following upon Mikulicz's paper of 1895 it appears that our view as regards the operative treatment of malignant growths of long bones has undergone a change. Whereas it has hitherto been generally accepted that especially in cases of periosteal sarcoma amputation or removal of the whole of the diseased bone is indicated, we now consider conservative treatment, consisting in excision of the tumour only, justifiable, and more so when an early stage of the growth is diagnosed. The $x$ rays have made such an early diagnosis almost the rule. There can be no doubt that Mikulicz was right when he maintained that patients suffering from such malignant growths, which as a rule give rise to little or no discomfort at all, will sooner undergo conservative treatment than sacrifice a limb. He is also right in saying that an early excision is more beneficial to the patient than a late amputation. Wherever the growth has produced secondary deposits, either in the bone itself or in the body, amputation gives no better results than excision. I am able to report on two cases of periosteal sarcoma of the tibia which I excised in 1900; both cases are doing well and show no signs of recurrence. The operative methods I used have, as far as I can see, been first applied by myself for malignant growths of the tibia. In my first case, that of a boy, aged 16 years, the growth was situated in the upper and middle thirds of the tibia. Without injuring the tumour or the periosteum of the tibia I excised a piece of the bone measuring about 30 centimetres. The stability of the limb was restored by a slight modification of a method which has been first described by Hahn in 1885 . After dividing the fibula immediately below its head I placed its pointed peripheral end into a bore-hole made in the upper epiphysis of the divided tibia. Ten weeks after the operation the fibula had become so firm in its new position that the patient was able to support the full weight of his body on the operated limb.

The accompanying radiographs (Figs. 11, 12, and 13) show the condition of the fibula in intervals of months and years after the operation. Now the functional hypertrophy of the fibula has reached such dimensions that the fibula is almost as thick as the tibia of the other and healthy side. Similar instances of furctional hypertrophy of the fibula have been recorded where the same method has been applied for extensive necrosis or traumatic defects of the tibia. It has been suggested to suture the lower end of the tibia to the transposed fibula but the radiographs of my case show that this procedure is unnecessary as the retraction of the interosseous membrane produces in itself the juxtaposition of the bones required.

In my second case the tumour invaded the lower twothirds of the tibia. In order to save the limb I removed a section of the fibula measuring almost the length of the excised part of the tibia. Now the tibial and fibular ends of the divided bone were respectively joined to one another by wire sutures. Complete recovery set in in five weeks and after five months the muscles, wbich at first were too long for the shortened limb, had adapted themselves to their altered function so perfectly that hardly any difference existed between the calf on the operated and the normal side. Such and similar operative methods which are well known to all of you enable the surgeon to save a limb even in cases where removal of almost the whole of a long bone is indicated. I only wish to refer to a case demonstrated to the German Congress of Surgeons in which the whole of the humerus was removed and the forearm fixed to the shoulder, the function of the hand thus being saved to the female patient who earned her living by needlework.

\section{Ligation OF LARGE VEINS.}

It is an extraordinary fact that in spite of the rapid advance of operative surgery within the last decade modern surgeons should have paid so little attention to research bearing on collateral circulation. Two important treatises on the subject have been published quite recently by Bier and Katzenstein. As yet research has almost exclu. sively been restricted to the various forms of collateral circulation following ligation of arteries. When we consider how long it took for surgeons to make up their minds to tie veins, especially the larger veins, of the body we understand more fully the apathy of surgeons towards the question of venous collateral circulation. Apart from the theoretical side of the question it is one of great practical importance as the domain of surgery has become wider and wider. In dealing with the radical cure of malignant growths the field of operation has become so extended that even the largest veins of the human body are brought under the surgeon's knife. The great advance in renal surgery has brought the vena cava and the question of its ligation into greater prominence. Scattered through literature we find several cases recorded in which the surgeon had accidentally injured the vein, tying it in one, suturing the wound of the vessel in the other, and merely using forcipressure again in another. Such cases have multiplied within the last two years, so that the time has arrived to consider more seriously how we should act when the nature of the operation necessitates injury of the vena cava. Anatomical, physiological, and pathological facts, as well as the results obtained by ligation of the vena cava in the human being, all prove that ligation is a legitimate procedure provided the ligature is placed below the influx of the renal veins. As in Bottini's, Zoege-Manteufel's, Heresco's, and Albarran's cases, I saw no evil consequences following the ligation of the vein which was necessitated by the excision of a tuberculous pyonephrotic kidney involving the vena cava. A few plates prepared in the following manner may demonstrate the vessels which establish collateral circulation. In a foetus I excised three centimetres of the vena cava, injected an emulsion of bismuthum and oil from a femoral vein and then radiographed successive phases of the injected specimen. As you see the pre-vertebral and intervertebral veins are the first to restore circulation.

It is an important fact first recorded by Israel that clinical symptoms may be absolutely absent in cases where the vena cava is involved or even obstructed by a diseased kidney. Therefore, it is necessary for the surgeon before excising a kidney to be prepared for a lesion of the vena cava. Although we should always give the retroperitoneal method the preference, yet whenever difficulties arise in treating the pedicle of the diseased organ the peritoneal cavity should be opened and the exact relation of the kidney to the vena cava should be determined ere the kidney is removed.

Ligation of the jugular, axillary, and even subclavian veins has been, and is being, so frequently performed that any further reference to such operations is unnecessary. Even simultaneous ligation of both common jugular veins remains without untoward consequences.

In a case of intrathoracic tuberculous goitre and another of secondary epipleural abscess following costal caries I have been obliged to tie the innominate vein; both operations were successful and no circulatory trouble ensued. My most remarkable experience has been the following. The patient was a woman, aged 34 years, who was suffering from chronic miliary tuberculosis. I traced its origin to a suppurating lymphatic gland which had invaded the bulb of the jugular vein. In excising the gland copious hæmorrhage resulted from a wound of the vein. Suture and lateral ligature were ineffective. Only after tying the common jugular, the subclavian, and innominate veins was I able to arrest the bleeding. Not the slightest circulatory trouble resulted. Two exactly similar operations have been recently published, with the same result even months after the operation. No abnormal veins were seen; circulation righted itself at once. It is a fact known to pathologists that even simultaneous thrombosis of the superior and inferior vena cava may leave the circulation unimpaired.

Anatomists such as Cruveilhier, Sappey, Poirier, and 